\title{
Fluorinated Alkoxide-Based Magnesium-Ion Battery Electrolytes that Demonstrate Li-Ion-Battery-Like High Anodic Stability and Solution Conductivity
}

\author{
Adam J. Crowe, Kyle K. Stringham, and Bart M. Bartlett ${ }^{*}$ \\ Department of Chemistry \\ University of Michigan \\ 930 N. University Avenue \\ Ann Arbor, MI 48109-1055, United States \\ Email:bartmb@umich.edu
}

Table of Contents

Figure S1. SEM image (top) and EDX spectrum (bottom) of deposited magnesium on S2 $\mathrm{Cu}$ foil from $1.2 \mathrm{M}\left(\left(\mathrm{CF}_{3}\right)_{2}\left(\mathrm{CH}_{3}\right)\right) \mathrm{COMgCl}$ and $0.2 \mathrm{M} \mathrm{AlCl}_{3}$ in THF electrolyte at $2 \mathrm{~mA}$ $\mathrm{cm}^{-2}\left(0.8 \mathrm{C} \mathrm{cm}^{-2}\right)$.

Figure S2. ${ }^{27} \mathrm{Al} \mathrm{NMR}$ chemical shifts for (a) $0.8 \mathrm{M}\left(\left(\mathrm{CF}_{3}\right)\left(\mathrm{CH}_{3}\right)_{2}\right) \mathrm{COMgCl}$ and $0.2 \mathrm{M}$ S3 $\mathrm{AlCl}_{3}$; (b) $1.2 \mathrm{M}\left(\left(\mathrm{CF}_{3}\right)_{2}\left(\mathrm{CH}_{3}\right)\right) \mathrm{COMgCl}$ and $0.2 \mathrm{M} \mathrm{AlCl}_{3}$; (c) $1.2 \mathrm{M}\left(\left(\mathrm{CF}_{3}\right)_{3}\right) \mathrm{COMgCl}$ and $0.2 \mathrm{M} \mathrm{AlCl}_{3}$ in THF.

Table S1. ${ }^{27} \mathrm{Al}$ NMR assignments of $\left(\left(\mathrm{CF}_{3}\right)_{x}\left(\mathrm{CH}_{3}\right)_{3-x}\right) \mathrm{COMgCl}(x=1,2$, and 3$)$ and $\mathrm{AlCl}_{3}$ solutions

Figure $\mathrm{S} 3 .{ }^{27} \mathrm{Al} \mathrm{NMR}$ chemical shifts for $y \mathrm{M}\left(\left(\mathrm{CF}_{3}\right)_{2}\left(\mathrm{CH}_{3}\right)\right) \mathrm{COMgCl}$ and $y / 6 \mathrm{M} \mathrm{AlCl} 3(y$ $=0.8,0.6$, and $0.4 \mathrm{M}$ ) in THF.

Table S2. ${ }^{27} \mathrm{Al} \mathrm{NMR}$ assignments for $y \mathrm{M}\left(\left(\mathrm{CF}_{3}\right)_{2}\left(\mathrm{CH}_{3}\right)\right) \mathrm{COMgCl}$ and $y / 6 \mathrm{M} \mathrm{AlCl} 3(y=$ $0.8,0.6$, and $0.4 \mathrm{M}$ ) in THF

Figure S4. ${ }^{27} \mathrm{Al} \mathrm{NMR}$ chemical shifts for $1.2 \mathrm{M}\left(\left(\mathrm{CF}_{3}\right)_{2}\left(\mathrm{CH}_{3}\right)\right) \mathrm{COMgCl}$ and $0.2 \mathrm{M} \mathrm{AlCl} 3 \quad \mathrm{~S} 5$ in $75 \% \mathrm{THF}$ and $25 \%$ 2-methyl-THF solution.

Table S3. ${ }^{27} \mathrm{Al} \mathrm{NMR}$ assignments for $1.2 \mathrm{M}\left(\left(\mathrm{CF}_{3}\right)_{2}\left(\mathrm{CH}_{3}\right)\right) \mathrm{COMgCl}$ and $0.2 \mathrm{M} \mathrm{AlCl}_{3}$ in S5 $75 \%$ THF and 25\% 2-methyl-THF solution. 

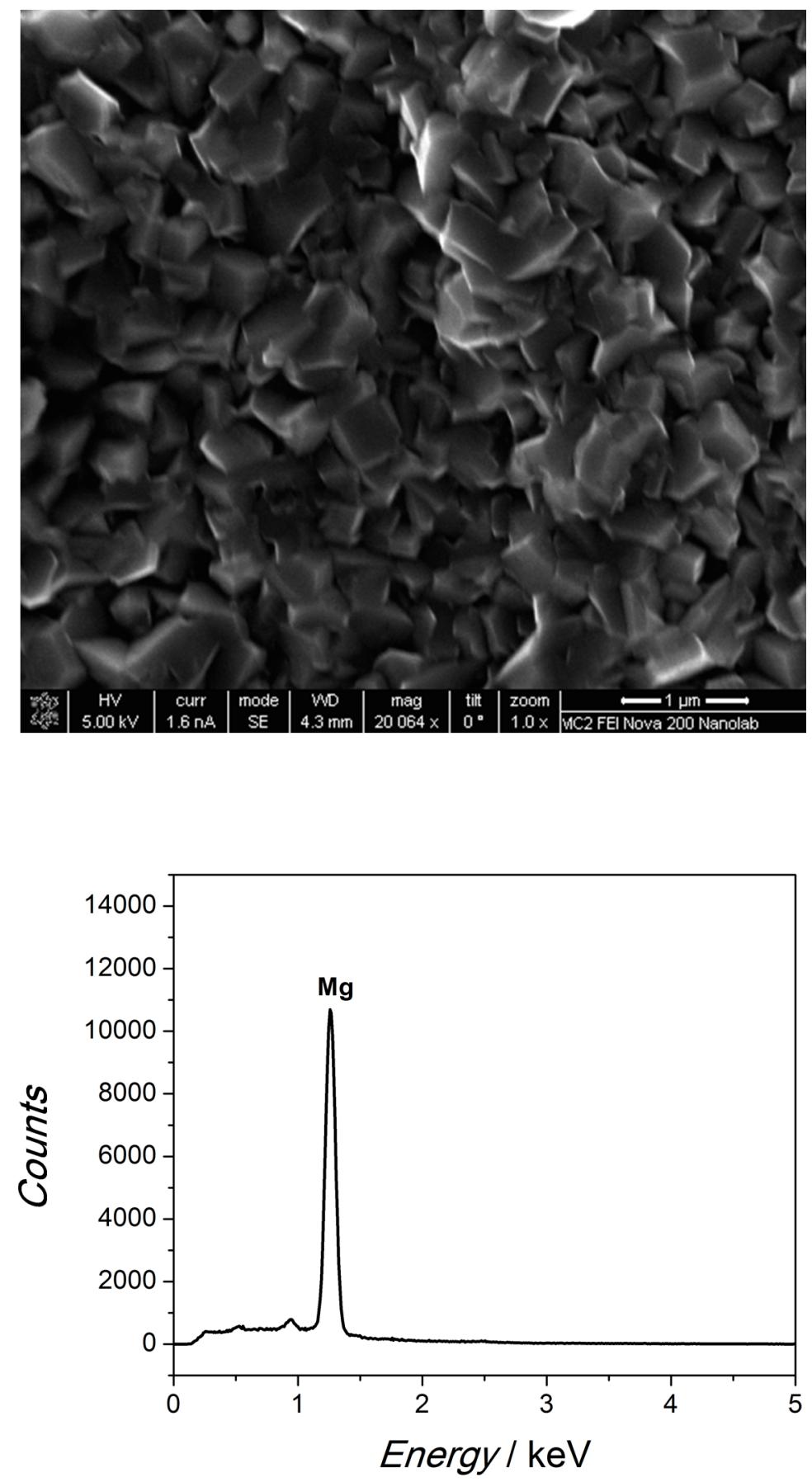

Figure S1. SEM image (top) and EDX spectrum (bottom) of deposited magnesium on $\mathrm{Cu}$ foil from $1.2 \mathrm{M}$ $\left(\left(\mathrm{CF}_{3}\right)_{2} \mathrm{CH}_{3}\right) \mathrm{COMgCl}$ and $0.2 \mathrm{M} \mathrm{AlCl}_{3}$ in THF electrolyte at $2 \mathrm{~mA} \mathrm{~cm}{ }^{-2}\left(0.8 \mathrm{C} \mathrm{cm}^{-2}\right)$. 
$\mathbf{a}$
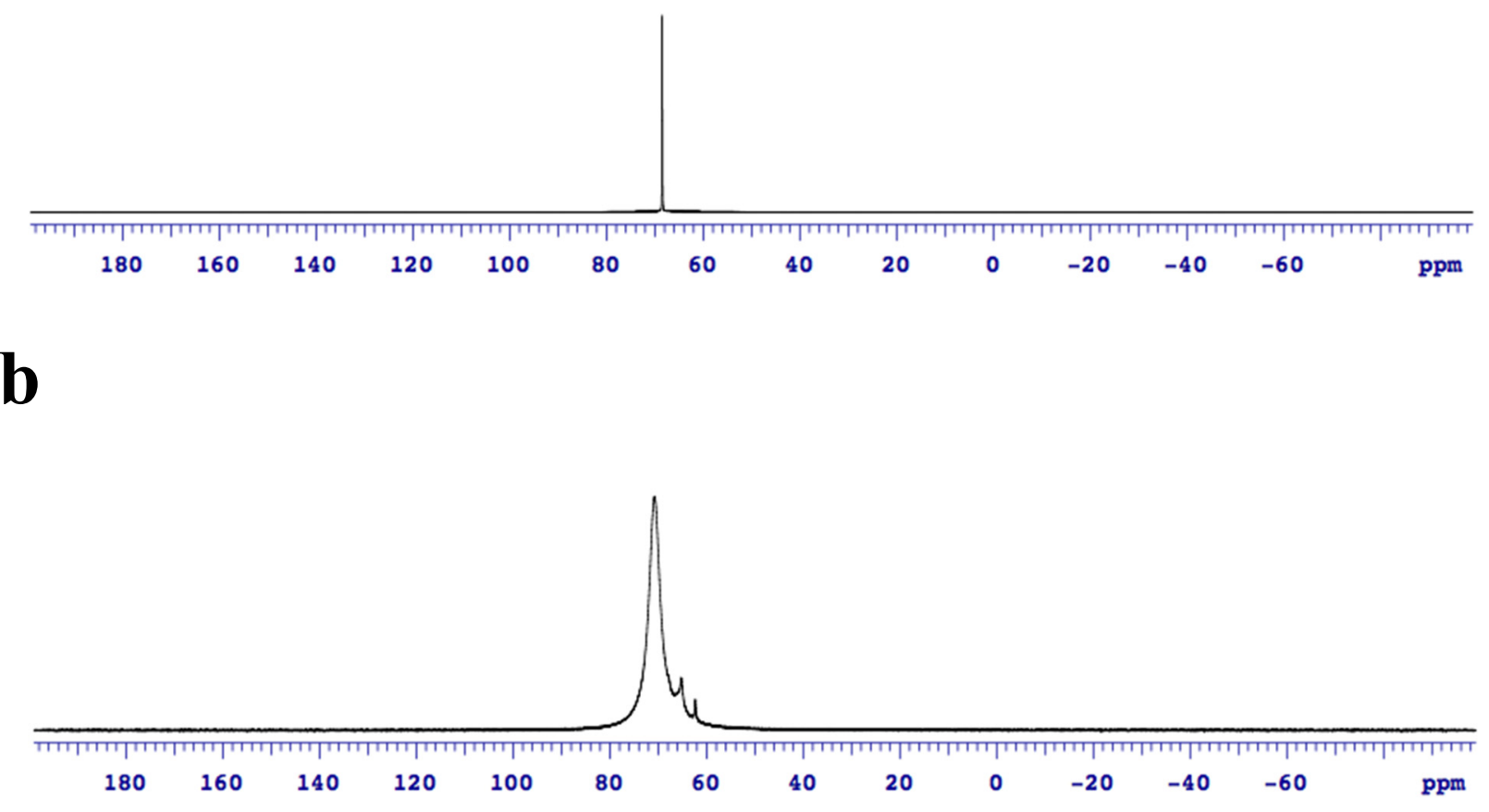

c

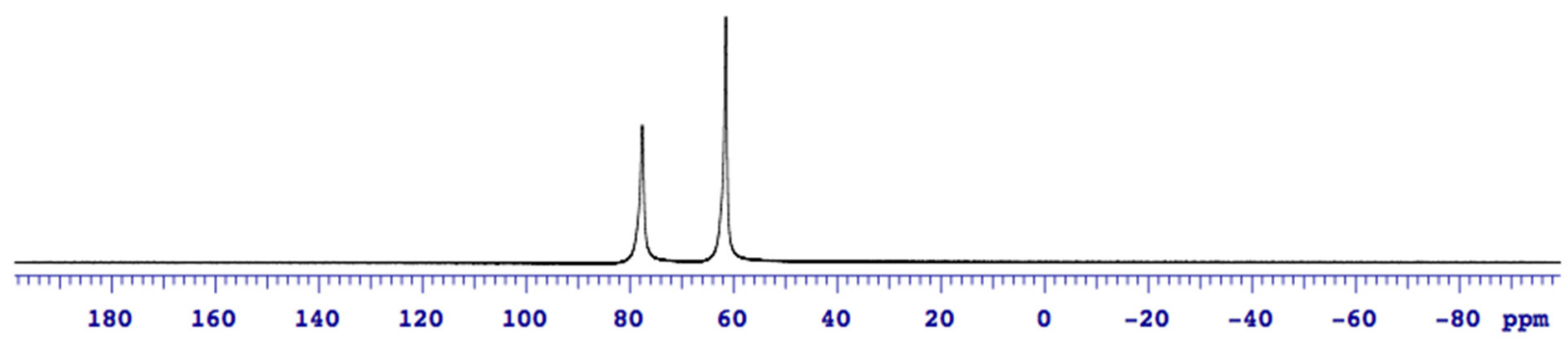

Figure S2. ${ }^{27} \mathrm{Al} \mathrm{NMR}$ chemical shifts for (a) $0.8 \mathrm{M}\left(\left(\mathrm{CF}_{3}\right)\left(\mathrm{CH}_{3}\right)_{2}\right) \mathrm{COMgCl}$ and $0.2 \mathrm{M} \mathrm{AlCl}_{3}$; (b) $1.2 \mathrm{M}$ $\left(\left(\mathrm{CF}_{3}\right)_{2}\left(\mathrm{CH}_{3}\right)\right) \mathrm{COMgCl}$ and $0.2 \mathrm{M} \mathrm{AlCl}_{3}$; (c) $1.2 \mathrm{M}\left(\left(\mathrm{CF}_{3}\right)_{3}\right) \mathrm{COMgCl}$ and $0.2 \mathrm{M} \mathrm{AlCl}_{3}$ in THF.

Table S1. ${ }^{27} \mathrm{Al} \mathrm{NMR}$ assignments of $\left(\left(\mathrm{CF}_{3}\right)_{x}\left(\mathrm{CH}_{3}\right)_{3-x}\right) \mathrm{COMgCl}(x=1,2$, and 3$)$ and $\mathrm{AlCl}_{3}$ solutions

\begin{tabular}{|c|c|c|}
\hline Electrolyte (R) & Chemical Shift / ppm & Assignment \\
\hline $0.8 \mathrm{M}\left(\mathrm{CF}_{3}\right)\left(\mathrm{CH}_{3}\right)_{2} \mathrm{COMgCl}$ and $0.2 \mathrm{M} \mathrm{AlCl}_{3}$ & 68 & {$\left[\left({ }^{\mathrm{R} 3} \mathrm{CO}\right)_{4} \mathrm{Al}\right]^{-}$} \\
\hline $1.2 \mathrm{M}\left(\mathrm{CF}_{3}\right)_{2} \mathrm{CH}_{3} \mathrm{COMgCl}$ and $0.2 \mathrm{M} \mathrm{AlCl}_{3}$ & $\begin{array}{l}71 \\
65 \\
62\end{array}$ & $\begin{array}{c}{\left[\left({ }^{\mathrm{R} 3} \mathrm{CO}\right)_{3} \mathrm{AlCl}\right]^{-}} \\
{\left[\left({ }^{\mathrm{R} 3} \mathrm{CO}\right)_{4} \mathrm{Al}\right]^{-}} \\
\mathrm{AlCl}_{3}\end{array}$ \\
\hline $1.2 \mathrm{M}\left(\mathrm{CF}_{3}\right)_{3} \mathrm{COMgCl}$ and $0.2 \mathrm{M} \mathrm{AlCl}_{3}$ & $\begin{array}{l}78 \\
61\end{array}$ & $\begin{array}{c}{\left[\left({ }^{\mathrm{R} 3} \mathrm{CO}\right)_{2} \mathrm{AlCl}_{2}\right]} \\
\left.\left[{ }^{\left({ }^{2} 3\right.} \mathrm{CO}\right)_{4} \mathrm{Al}\right]^{-}\end{array}$ \\
\hline
\end{tabular}




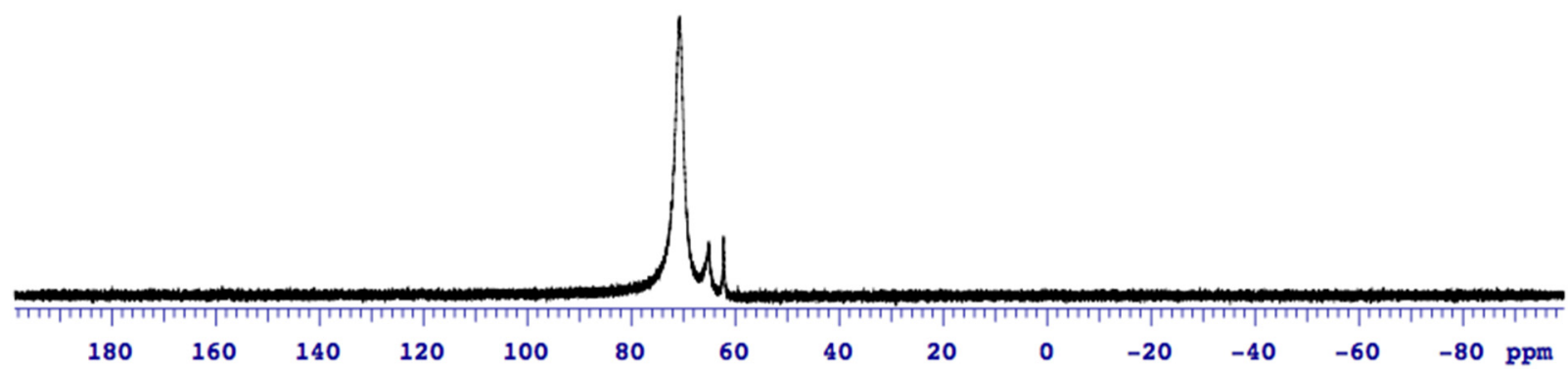

Figure S3. ${ }^{27} \mathrm{Al} \mathrm{NMR}$ chemical shifts for $y \mathrm{M}\left(\left(\mathrm{CF}_{3}\right)_{2}\left(\mathrm{CH}_{3}\right)\right) \mathrm{COMgCl}$ and $y / 6 \mathrm{M} \mathrm{AlCl}_{3}(y=0.8,0.6$, and $0.4 \mathrm{M}$ ) in THF.

Table S2. ${ }^{27} \mathrm{Al} \mathrm{NMR}$ assignments for $y \mathrm{M}\left(\left(\mathrm{CF}_{3}\right)_{2}\left(\mathrm{CH}_{3}\right)\right) \mathrm{COMgCl}$ and $y / 6 \mathrm{M} \mathrm{AlCl}_{3}(y=0.8,0.6$, and 0.4 $\mathrm{M})$ in THF.

\begin{tabular}{ccc}
\hline Electrolyte $(\mathrm{R})$ & Chemical Shift $/ \mathrm{ppm}$ & Assignment \\
\hline $\left.0.80 \mathrm{M}\left(\left(\mathrm{CF}_{3}\right)_{2} \mathrm{CH}_{3} \mathrm{COMgCl}\right)\right)$ and $0.13 \mathrm{M}$ & 71 & {$\left[\left({ }^{\mathrm{R} 3} \mathrm{CO}\right)_{4} \mathrm{Al}\right]^{-}$} \\
$\mathrm{AlCl}_{3}$ & 65 & {$\left[\left({ }^{\mathrm{R} 3} \mathrm{CO}\right)_{3} \mathrm{AlCl}\right]^{-}$} \\
& 62 & $\mathrm{AlCl}_{3}$ \\
$0.60 \mathrm{M}\left(\left(\mathrm{CF}_{3}\right)_{2} \mathrm{CH}_{3} \mathrm{COMgCl}\right)$ and $0.10 \mathrm{M}$ & 71 & {$\left[\left({ }^{\mathrm{R} 3} \mathrm{CO}\right)_{4} \mathrm{Al}\right]^{-}$} \\
$\mathrm{AlCl}_{3}$ & 65 & {$\left[\left({ }^{\mathrm{R} 3} \mathrm{CO}\right)_{3} \mathrm{AlCl}\right]^{-}$} \\
& 62 & $\mathrm{AlCl}_{3}$ \\
$0.40 \mathrm{M}\left(\left(\mathrm{CF}_{3}\right)_{2} \mathrm{CH}_{3} \mathrm{COMgCl}\right)$ and $0.07 \mathrm{M}$ & 71 & {$\left[\left({ }^{\mathrm{R} 3} \mathrm{CO}\right)_{4} \mathrm{Al}\right]^{-}$} \\
$\mathrm{AlCl}$ & 65 & {$\left[\left({ }^{\mathrm{R} 3} \mathrm{CO}\right)_{3} \mathrm{AlCl}\right]^{-}$} \\
& 62 & $\mathrm{AlCl}_{3}$ \\
\hline
\end{tabular}




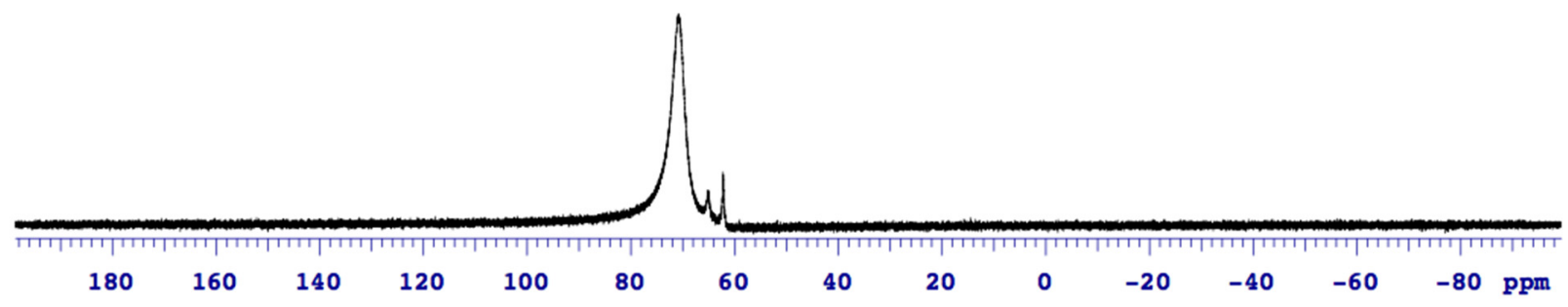

Figure S4. ${ }^{27} \mathrm{Al} \mathrm{NMR}$ chemical shifts for $1.2 \mathrm{M}\left(\left(\mathrm{CF}_{3}\right)_{2}\left(\mathrm{CH}_{3}\right)\right) \mathrm{COMgCl}$ and $0.2 \mathrm{M} \mathrm{AlCl}$ in $75 \%$ THF and 25\% 2-methyl-THF solution.

Table S3. ${ }^{27} \mathrm{Al} \mathrm{NMR}$ assignments for $1.2 \mathrm{M}\left(\left(\mathrm{CF}_{3}\right)_{2}\left(\mathrm{CH}_{3}\right)\right) \mathrm{COMgCl}$ and $0.2 \mathrm{M} \mathrm{AlCl}_{3}$ in $75 \% \mathrm{THF}$ and 25\% 2-methyl-THF solution.

\begin{tabular}{ccc}
\hline Electrolyte (R) & Chemical Shift / ppm & Assignment \\
\hline $0.8 \mathrm{M}\left(\mathrm{CF}_{3}\right)_{2} \mathrm{CH}_{3} \mathrm{COMgCl}$ and $0.2 \mathrm{M} \mathrm{AlCl}_{3}$ & 71 & {$\left[\left({ }^{\mathrm{R} 3} \mathrm{CO}\right)_{4} \mathrm{Al}\right]^{-}$} \\
& 65 & {$\left[\left({ }^{\mathrm{R} 3} \mathrm{CO}\right)_{3} \mathrm{AlCl}\right]^{-}$} \\
& 62 & $\mathrm{AlCl}_{3}$ \\
\hline
\end{tabular}

